\title{
Assessment of post-operative pain control using convential parenteral route versus patient controlled analgesia
}

\begin{abstract}
Pain management following surgical interventions is a crucial for patient satisfaction and rapid recovery. Therefore, models to improve patient pain outcomes will achieve these goals. Patient controlled analgesia (PCA) is an alternative method used to control different types of pain compared to other routes. The present study was aimed to assess postoperative pain intensity in patients after surgical interventions using either parenteral pain killers or an intravenous PCA. In this study, one hundred and sixty eight patients who underwent different surgical operations were divided into six groups with a subsequent PCA group. After pain intensity was evaluated, each patient received either pethidine, diclofenac sodium or paracetamol (drug analgesic therapy) one hour after recovery from anesthesia. Time from the initiation of treatment to the request for analgesics was measured and represents the effectiveness of the analgesic drug or PCA treatments. In addition, the total medications intake was recorded during the first 12 postoperativehours. Our findings showed that postoperative pain scores at 1 hour and 12 hours after surgery were significantly $(p<0.05)$ lower among all the patients compared with pre-analgesic treatments. Furthermore, in the PCA patients group, the pain scores were lower in major surgery with more patients satisfaction compared with other treated groups $(p<0.05)$. Overall, pain-killer administration was higher in the six groups but this difference was found to be not statistically significant compared with intravenous PCA group. Thus, it is concluded that intravenous PCA model is an effective approach that can manage acute postoperative pain and improve patient satisfaction.
\end{abstract}

Keywords: postoperative pain, pain score, analgesia, patient, analgesic
Volume 4 Issue I - 2016

Nasruddin E EL-Reyani, Nowara M Abdsalam,
Wafa S Sulaiman, Fathi M Sherif

Department of Pharmacology and Clinical Pharmacy, Libya

Correspondence: Nasruddin E EL-Reyani, Department of Pharmacology and Clinical Pharmacy, Faculty of Pharmacy, University of Tripoli, Tripoli, Libya, Tel 00218 21 462 7798, Email nelreyani@gmail.com

Received: December 0I, 2015 | Published: January 28, 2016

\section{Introduction}

Pain control during postoperative stage is vital for patient satisfaction and rapid recovery. Although, many pain killers were used to control pain symptoms, self-administered medications using patient controlled analgesia (PCA) can provide an alternative route to controlled postoperative pain control progress. It has previously been reported that PCA is preferred when regional analgesia is unavailable, contraindicated or when less invasive drug administration routes are used by the surgeon. ${ }^{1}$ Some studies have shown an optimal pain control when PCA was used postoperatively with different medications. ${ }^{1,2}$ Other studies, however, have shown that epidural analgesia still recommended to be superior analgesia to systemic opioid and PCA for different patients. ${ }^{3,4}$ This lack of consensus on the appropriate method use of analgesia has ranked intravenous-PCA (IVPCA) as standard practice for postoperative pain control at various hospitals. ${ }^{5}$ Moreover, IV-PCA was reported to be a superior method with high patient satisfaction scores. ${ }^{6,7}$ Although PCA has been used for more than four decades, at a domestic level, it is not commonly used. To the best of our knowledge, this study is the first to show the effects of PCA in managing postoperative pain (using fentanyl) at a single tertiary hospital, Abusleem Trauma Center. Moreover, other analgesic methods are employed at other two tertiary hospitals; Ali Askar Neurosurgery Center and Aljala Hospital for Gynecology and Obstetrics at Tripoli city in Libya. Indeed, the present study was aimed to investigate the effectiveness of PCA in patients who underwent major surgical interventions and compared them with others using different pain relief drugs and to report on patient satisfaction and outcomes.

\section{Methods}

We did perform a search strategy in a purpose to identify patients undergone post-operative pain management using different analgesia. Our basic search was based on other observations concerning the potential role of PCA.

\section{Inclusion and exclusion criteria}

Patients enrolled in this study were selected from two different teaching hospitals; Neurology and Neurosurgery and Gynecology and Obstetrics hospitals, and one general hospital; emergency intervention and trauma hospital. The inclusion criteria were patients devoid from pre-operative surgical intervention for the last three weeks, and those underwent surgical either minor or major day case intervention in the last two weeks. In addition, patients who are more than eighteenyears old, have immediate surgical intervention, elective surgery procedure and case-indicated procedures as those seen in Gynecology ward. The exclusion criteria were patients who used over the counter (OTC) drug intentionally for pain relief prior to any day-case procedure. Diabetic patients on insulin therapy were also excluded. Regional blocks, continuous use of analgesics, uncontrolled hypertension and contraindications limited the use of PCA were considered factors for excluding the participants. 


\section{Research design}

For the purpose of the study, the search on the pain and postoperative analgesia and PCA was assessed using electronic engine for search. The overall available evidence for post-operative analgesia was compared and reassessed. It is also looked for studies primarily compared the effect of different types of analgesia and patient satisfaction with PCA. For measuring patient satisfaction, we used a pre-modified patient satisfaction questionnaires; PSQ-18 to meet the criterion of domestic evaluation. ${ }^{8}$ Such an instrument has the advantage of good reliability and validity. ${ }^{9}$

\section{Patient cohorts}

One hundred and seventy seven patients were primarily recruited to either PCA or nurse controlled analgesia (NCA). Nine patients were under 18years old and so were excluded from the study. The rest (168 patients) underwent six types of surgical interventions inducing mild, moderate and severe post-operative pain. After verbal consent, but before the onset of active procedure, was obtained a signed consent has also been received from each patient. The terms of research were explained personally and the available data were individually interviewed, examined, investigated and treated as per study protocol confidentially. The treatment regimens were opioid and non-steroidal anti-inflammatory drugs. Each patient has postoperative pain intensity prior to trial medication been evaluated. Analgesia after surgery was given as requested to all the patients using different brands of pain killers.

\section{Procedure}

This study was carried out during the period of six months, from June to November. The pain score intensity was measured in every patient. Prior to surgical intervention, all patients were asked to read the instruction paper along with the use of a verbal rating scale for the measurement of postoperative pain. We practically involved interview with patients at the night of surgical procedure to explain how to determine pain scores at the commencement of recovery. A separate bed-side attached questionnaire including personal data, present condition of the patient, type of surgery and data regarding analgesic drugs and their duration were used to assess the frequency of pain score. Patients were asked to answer the questions related to their evaluation either personally or by the aid of their relatives. A score number $(0-10)$ was given to assess the intensity of pain score, where 0 represented no pain, 1-3 represented mild pain interfering little with daily activity (nagging), 4-6 moderate pain that interferes significantly with daily activity, and 7-10 represented severe pain that causes disabling and make patient unable to perform daily activity. According to pain scores physician in charge and nurse can determine the type of analgesia that patient require. Furthermore, to measure the level of satisfaction patients were asked to rank their satisfaction towards their pain management within 12 hours after receiving drug therapy or PCA according to a scale designed for assessment from 0 , represented unsatisfaction to 5 that represented the optimum satisfaction. All postoperative assessments were done under the supervision of under-training clinical pharmacist who did participate in conducting this study. In the PCA group, patients were instructed on the use of PCA pump and an additional instruction guidelines was delivered to them for further reading attention.

\section{Treatments}

Approximately, one hour after recovery, either NCA or PCA was instituted in patients who had no analgesics in the recovery room. An hour rest interval was followed to avoid cross administrations of different analgesics. PCA patients were free to request further analgesia, if necessary, according to PCA procedure. In NCA, the analgesic medications namely pethidine, diclofenac sodium and paracetamol were different in the six patient cohorts. Patients could request further analgesia during the first 12 hours after operation, and the same doses were administered at each request. The time from the beginning of treatment to the request of another dose was recorded and represented the effectiveness of the drugs and PCA treatments. In addition, the total medication intake was recorded during the first 12 postoperativehours. Initial pain intensity "before treatment" was assessed by a numeric rating scale from 0 (no pain) to 10 (unbearable pain).

\section{Protocol and data collection}

Since the files included in this study reported the course and efficacy of drug therapy with different measurements, and since therapeutic options also varied between files. Therefore, we distributed participants according to surgical intervention into neurosurgery, orthopedic surgery, gynecology surgery, general surgery and urinary surgery. Moreover, a separate group was investigated using PCA.

\section{Ethics}

The study design was complied with rules and regulations of guidelines for domestic clinical trials. The protocol was approved by the Ethics Committee (Department of Pharmacology and Clinical Pharmacy, Faculty of Pharmacy, University of Tripoli, Hospitals Supreme Committees and the National Component Authority, Libyan National Authority for Scientific Research).

\section{Statistical analysis}

The differences between and within treatment groups were analyzed by the use of one-way ANOVA followed by the use of post hoc Tukey's test for multiple comparisons. Pain intensity was also analyzed taking into account the pain interval. Data was analyzed using Student $t$-test and Mann-Whitney $U$ test, as appropriate. Values are presented as the mean $\pm \mathrm{SD}$. All analyses were performed using SPSS for Windows version 20.0 (IBM SPSS Inc., Chicago, IL, USA). Differences were considered to be statistically significant at $p<0.05$.

\section{Results}

Patients' characteristics including age, body weight along with surgical procedures are shown in Table 1. One hundred and sixty eight patients were completed the study. While all the patients (100\%) displayed post-operative pain score of 10 , the pain intensity was not statistically different between the patients among all the groups preoperatively. Nevertheless, the six types of surgical procedures produced different intensities of post-operative pain. The cohort groups have experienced severe pain with a numeric rating score of 8 to 10 , while the cohorts of less severity interventions had moderate to severe pain with most scores of 6 to9. In contrast, the day case surgery cohort group has experienced only mild to moderate pain with a score of 4-6. No differences in pain intensity scores were observed between the later treatment groups in each patient cohort.

In general and according to the pain score, the present study indicated that the ranked drug used in controlling post-operative pain was pethidine at $81.5 \%$, followed by diclofenac sodium at $18.5 \%$ and paracetamol at $15.7 \%$ (Figure 1). PCA group used only fentanyl for $75 \%$ of the patients. In groups with pain score ranging from 6 to 9 ; gynecology surgery $(100 \%)$, orthopedic surgery $(82.35 \%)$ and general surgery group patients $(89.23 \%)$ showed significant relief of pain with parenteral pethidine use $(1.4 \pm 0.11,1.2 \pm 0.1$ and $1.5 \pm 0.1$ 
respectively against the control value $7.6 \pm 0.11, p<0.05$, Figure 2). Moreover, in genito-urinary surgery, 13 patients $(81 \%)$ displayed significant reduction in the pain score after using diclofenac sodium or paracetamol $(2.3 \pm 0.13$ and $3.4 \pm 0.42$ respectively against the control, $7.1 \pm 0.21, p<0.05$, Figure 3 ). In neurosurgery, the most common postoperative analgesic drug used was pethidine per se or combined with diclofenac sodium.

Table I Patients characteristics, pain intensity and surgical data unless PCA is stated, pethidine was the main analgesic drug used

\begin{tabular}{|c|c|c|c|c|}
\hline \multirow[b]{2}{*}{ Age(years) } & \multicolumn{4}{|c|}{ Patients $(n=\mid 68)$ Mean $\pm S D$} \\
\hline & $37.9 \pm 1.2$ & & & \\
\hline Weight(kg) & $77.9 \pm 0.7$ & & & \\
\hline \multirow{2}{*}{$\begin{array}{l}\text { Surgical } \\
\text { procedures }\end{array}$} & \multicolumn{4}{|l|}{ Pain score } \\
\hline & $\begin{array}{l}\text { No. of } \\
\text { patients }\end{array}$ & $\begin{array}{l}\text { pre- } \\
\text { operative }\end{array}$ & $\begin{array}{l}\text { post- } \\
\text { operative }\end{array}$ & p-value \\
\hline Orthopedic & 33 & $7.2 \pm 0.2$ & $1.15 \pm 0.1 * *$ & $<0.01$ \\
\hline Head and neck & 3 & $8.3 \pm 1.9$ & $1.3 \pm 0.7 * *$ & $<0.01$ \\
\hline Open abdomen & 46 & $7.0 \pm 0.1$ & $1.5 \pm 0.1 * *$ & $<0.01$ \\
\hline $\begin{array}{l}\text { Thoracic } \\
\text { "Fentanyl (PCA)" }\end{array}$ & 9 & $7.0 \pm 0.3$ & $1.3 \pm 0.2^{* *}$ & $<0.01$ \\
\hline Genito-urologic & 13 & $7.1 \pm 0.2$ & $1.7 \pm 0.1 * *$ & $<0.01$ \\
\hline Gynecology & 64 & $7.6 \pm 0.1$ & $1.4 \pm 0.1 * *$ & $<0.01$ \\
\hline All patients & 168 & $7.6 \pm 0.1$ & $1.4 \pm 0.1 * *$ & $<0.01$ \\
\hline
\end{tabular}

** Statistically significant different at $\mathrm{P}<0.01$

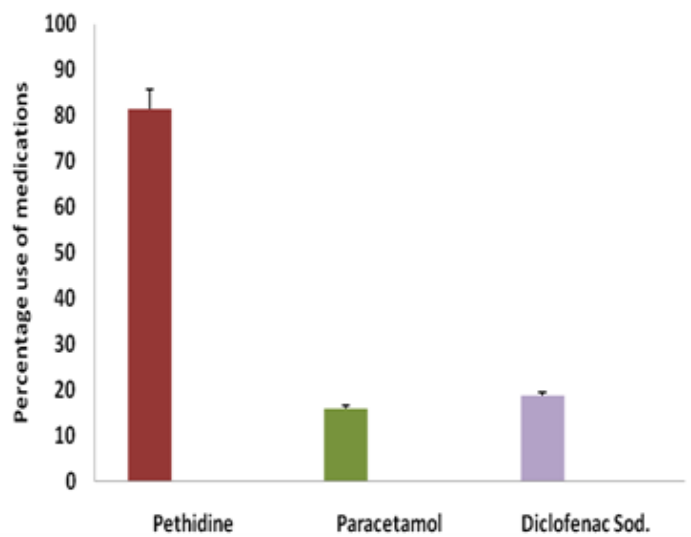

Figure I Percentage use of pethidine, diclofenac sodium and paracetamol after post-operative pain.

** Statistically significant different at $\mathrm{P}<0.01$.

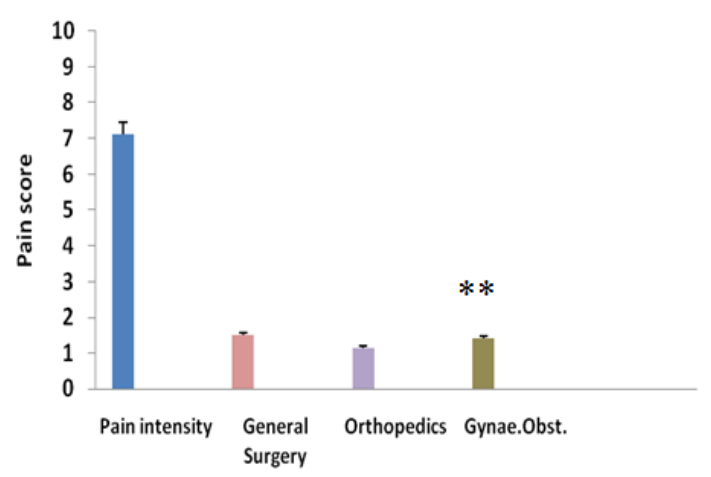

Figure 2 Use of pethidine in different surgical procedures.

*Statistically significant different at $\mathrm{P}<0.05$

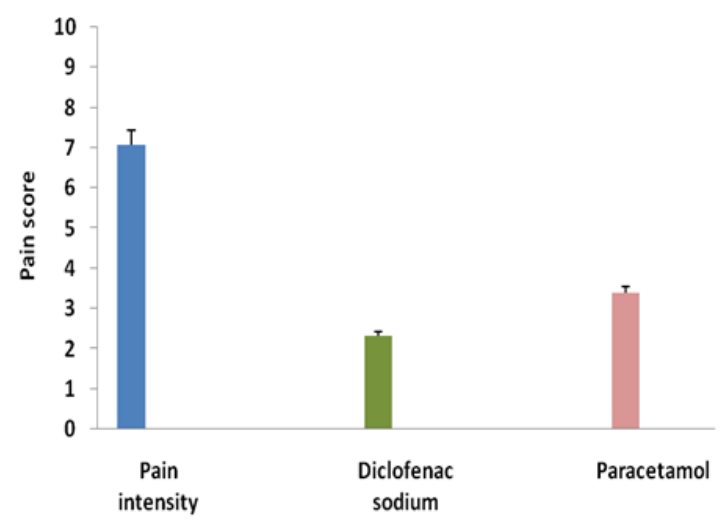

Figure 3 Effects of diclofenac sodium and paracetamol on post-operative genito-urinary pain intensity.

*Statistically significant different at $\mathrm{P}<0.05$.

On the other hand, the use of fentanyl in PCA produced significant important impact in thoracic surgery procedures in terms of reducing pain intensity ( $1.3 \pm 0.2$ against the control $7.0 \pm 0.3, p<0.05$, Figure 4$)$. Moreover, PCA has resulted in more patient satisfaction compared with other routes of administration (data not shown). The present study showed significant impact on the use of pethidine as postoperative analgesic compared to the least analgesic drugs used diclofenac sodium and paracetamol $(P<0.05)$ (Figure 1) (Figure 2). In terms of patient satisfaction, all the patients underwent postoperative IV-PCA showed significant satisfaction compared with other groups (data not shown). The reason for dissatisfaction was obviously attributed to continuous complaints of patients on the needs for pain killers.

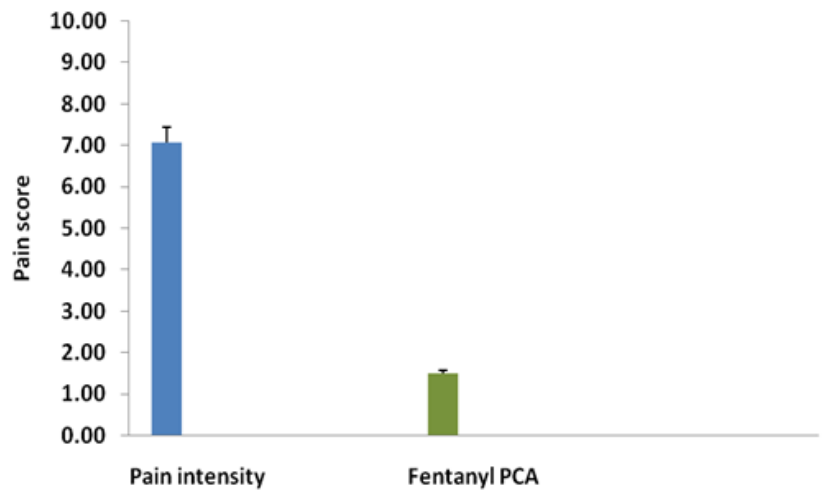

Figure 4 Effect of fentanyl IV-PCA on post-operative pain intensity. *Statistically significant different at $\mathrm{P}<0.05$.

\section{Discussion}

Although IV-PCA was used over other methods for postoperative pain control, there is still a considerable amount of debate over whether or not to offer an additional comfort and satisfaction to patients. Accordingly, the use of IV-PCA has been discouraged by a number of studies, ${ }^{10}$ although some found otherwise. ${ }^{11,12}$ The employment of IVPCA with the intention of achieving optimum effects has been studied on a small scale with inconsistent result. ${ }^{13,14}$ Therefore, the present prospective study was conducted to elucidate the effectiveness of IV-PCA during the first 12 hours after major surgical procedure and to understand whether PCA is a useful tool in the control of acute postoperative pain. The data obtained from patients of this study helped us to formulate some guidelines for the use of PCA in major surgeries. 
The present study showed that background infusion rate of fentanyl was being used for IV-PCA without causing severe adverse effects. Most importantly, adding both an adjuvant analgesic and an antiemetic to the IV-PCA regimen was found to simultaneously decrease the background infusion rate required for effective pain relief as well as the incidence of postoperative nausea and vomiting. Typically, the strongest, most frequent and most painful postoperative pain occurs by the end of the surgical procedure as the muscles regain their relaxation status. While the vast majority of patients described at least some stages of pain as painful, the severity of reported pain varies considerably. Several factors have been shown to intensify pain experienced by patients after surgeries. These include continuous demand of analgesic drugs and lack of attendance of caregivers to respond to the demand..$^{15}$ The other key determinant that may influence the pain level that a patient experiences is lack of a satisfactory method in controlling pain. ${ }^{16}$ Therefore, having a method for controlling pain without prolonging the cycle of demand will foster a patient's sense of self-management and therefore, confidence in his capacity to control pain and perception.

To the best of our knowledge, this study is the first to compare the use of IV-PCA with traditional use of other parenteral pain killers at domestic level. This new model of controlling pain in patients underwent major surgical procedures is a method in which the patient can modify the pain intensity by means of controlling pain without the need for health professionals at a range keeps the drug's use safe. Parenteral pain killer administration is the most commonly method used in post-operative pain control for patients undergoing different major surgeries. However, despite its narrow use, our findings showed that PCA was more effective in the control of acute post-operative moderate to severe pain. PCA was also significantly more effective during the 12 hours post-surgery. Moreover, the efficiency of PCA in controlling pain intensity can be seen in the set of acute major surgery patients as well. This contradicts other study that showed the limited use of PCA during the postoperative acute phase due to various side effects of narcotics. ${ }^{17}$ However, if there are minimal side effects, IV-PCA can be used in accordance with additional medication such as anti-emetics. The second point of this study was to evaluate the patient satisfaction. All studies conducted are agreed on the impact of measuring patient satisfaction on quality improvement of care. Patients' involvement of measuring satisfaction is a realistic tool to provide opportunity for improvement, enhance strategic decision making, reduce cost, meet patients' expectations, frame strategies for effective management, monitor healthcare performance of health plans and provide benchmarking across the healthcare institutions. ${ }^{18,19}$ Basically, there are two approaches for evaluating patient satisfaction, qualitative and quantitative. The quantitative approach provides accurate methods to measure patient satisfaction. Standardized questionnaires, either self-reported or interviewer-administrated, were the most common assessment tool for conducting patient satisfaction studies. ${ }^{9,19-21}$ In terms of measuring the impact of patient satisfaction using a number of analgesic drugs on post-operative periods and compare them with the used PCA, it has been seen that the intermittent pain that the patient experienced while requesting traditional administration of different analgesics has resulted in more time till receiving the agent. In PCA group, the overall patients' satisfaction scores on the management of analgesia did differ significantly compared with other groups. In terms of the number of times a patient administered additional analgesia, on average the parenteral group administered analgesia was more frequently than the
PCA group. The dissatisfaction seen in other groups was reasoned to continuous demand and staff calls to get their drugs being given. These findings were in coincidence with others observations that mistrust and dissatisfaction on the part of the patients, when combined, creates formidable obstacles to successful treatment of pain..$^{15,10}$ Moreover, despite that only fentanyl was used as IV-PCA, the development of postoperative nausea and vomiting was only seen with pethidine. This might be attributed to the small number of patients receiving fentanyl without ruling out its side effects. Nevertheless, the use of PCA is restricted in our hospitals, however, on practical bases as well as theoretical ones as in many busy hospital words, staff numbers and time may serve to limit the efficiency of conventional method and PCA will remain a commonly used method of analgesia. More intensive and extensive study protocol is needed to draw a basic guideline in controlling post-operative pain under circumstances that meet the use of PCA. It is generally postulated that post-operative pain is arguably the most common clinical problem in hospitals, it is often dismissed with an order for intermittent intramuscular opiate injections given at the discretion of an over worked nursing staff. This generally results in patients waiting for pain relief, then a period of relief and perhaps drowsiness and then the cycle is repeated. With this method, pain relief is only satisfactory for about one-third of the time; this makes the patient always complain that the nurse did not give him analgesic after surgery.

\section{Conclusion}

The present study concluded that the field of post-operative pain management is being given more attention as essential component of the care of surgical patients; the overriding principle of post-operative pain management is to provide general background of analgesia that sufficient to relief pain. The most common method of postoperative pain relief is the use of 'on demand' intramuscular opioid injections. Better pain relief can be obtained with newer techniques such as epidural opioid and PCA. The later may offer more satisfactory results that both physicians and patients seeking for.

\section{Acknowledgements}

None.

\section{Conflict of interest}

Author declares that there is no conflict of interest.

\section{References}

1. Palmer PP, Miller RD. Current and developing methods of patient-controlled analgesia. Anesthesiol Clin. 2010;28(4):587-599.

2. Butkovic D, Kralik S, Matolic M, et al. Postoperative analgesia with intravenous fentanyl PCA vs epidural block after thoracoscopicpectus excavatum repair in children. Br J Anaesth. 2007;98(5):677-681.

3. Rugyte DC, Kilda A, Karbonskiene A, et al. Systemic postoperative pain management following minimally invasive pectus excavatum repair in children and adolescents: a retrospective comparison of intravenous patient-controlled analgesia and continuous infusion with morphine. Pediatr Surg Int. 2010;26(7):665-669.

4. Volmanen P, Sarvela J, Akural EI, et al. Intravenous remifentanil vs. epidural levobupivacaine with fentanyl for pain relief in early labour: a randomised, controlled, double-blinded study. Acta Anaesthesiol Scand. 2008;52(2):249-255. 
5. Shin S, Min KT, Shin YS, et al. Finding the 'Ideal' regimen for fentanyl-based intravenous patient-controlled analgesia: how to give and what to mix? Yonsei Med J. 2014;55(3):800-806.

6. Ballantyne JC, Carr DB, Chalmers TC, et al. Postoperative patient-controlled analgesia: meta-analyses of initial randomized control trials. $J$ Clin Anesth. 1993;5(3):182-193.

7. Walder B, Schafer M, Henzi I, et al. Efficacy and safety of patient-controlled opioid analgesia for acute postoperative pain. A quantitative systematic review. Acta Anaesthesiol Scand. 2001;45(7):795-804.

8. Aerlyn GD, Paul PL. Patient satisfaction instruments used at academic medical centers: results of a survey. Amer J Med Qual. 2003;18(6):265-269.

9. Urden LD (2002) Patient satisfaction measurement: current issues and implications. Outcomes Manag. 2002;6(3):125-131.

10. Macintyre PE. Safety and efficacy of patient-controlled analgesia. $\mathrm{Br} \mathrm{J}$ Anaesth. 2001;87(1):36-46.

11. Guler T, Unlugenc H, Gundogan Z, et al. A background infusion of morphine enhances patient-controlled analgesia after cardiac surgery. Can J Anaesth. 2004;51(7):718-722.

12. White I, Ghinea R, Avital S, et al. Morphine at "sub-analgesic" background infusion rate plus low-dose PCA bolus control pain better and is as safe as twice a bolus-only PCA regimen: a randomized, double blind study. Pharmacol Res. 2012;66(2):185-191.

13. White PF, Kehlet H. Improving postoperative pain management: what are the unresolved issues? Anesthesiology. 2010;112(1):220-225.
14. Wu CL, Raja SN. Treatment of acute postoperative pain. Lancet. 2011;377(9784):2215-2225.

15. Motov SM, Khan AN. Problems and barriers of pain management in the emergency department: Are we ever going to get better? J Pain Res. 2009;2:5-11.

16. Asgari MM, Warton ME, Neugebauer R, et al. Predictors of patient satisfaction with Mohs surgery. Analysis of preoperative, intraoperative, and postoperative factors in a prospective cohort. Arch Dermatol. 2011;147(12):1387-1394.

17. White PF. The role of non-opioid analgesic techniques in the management of pain after ambulatory surgery. Anesth Analg. 2002;101(Suppl):S5-22.

18. Cheng SH, Yang MC, Chiang TL. Patient satisfaction with and recommendation of a hospital: effects of interpersonal and technical aspects of hospital care. Int J Qual Health Care. 2003;15(4):345-355.

19. Bjertnaes OA, Sjetne IS, Iversen HH. Overall patient satisfaction with hospitals: effects of patient-reported experiences and fulfillment of expectations. BMJ Qual Saf. 2012;21(1):39-46.

20. Quintana JM, Gonzalez N, Bibbao A, et al. Predictors of patien satisfaction with hospital health care. BMC Health Serv Res. 2006;6:102

21. Wilder-Smith OH, Möhrle JJ, Martin NC. Acute pain management after surgery or in the emergency room in Switzerland: a comparative survey of Swiss anaesthesiologists and surgeons. Eur J Pain. 2002;6(3):189-201. 